Sociologie et sociétés

\title{
La rébellion des sans-visage
}

Analyse de l'émergence du mouvement zapatiste au Chiapas (Mexique)

\section{The Rebellion of the Faceless.}

\section{Analysis of the Rise of the Zapatist Movement in the Chiapas (Mexico)}

\section{Gilles Cormier}

Volume 36, numéro 1, printemps 2004

Goûts, pratiques culturelles et inégalités sociales : branchés et exclus Tastes, Cultural Practices and Social Inequalities: In Fashion and Out

URI : https://id.erudit.org/iderudit/009590ar

DOI : https://doi.org/10.7202/009590ar

Aller au sommaire du numéro

Éditeur(s)

Les Presses de l'Université de Montréal

ISSN

0038-030X (imprimé)

1492-1375 (numérique)

Découvrir la revue

Citer cet article

Cormier, G. (2004). La rébellion des sans-visage : analyse de l'émergence du mouvement zapatiste au Chiapas (Mexique). Sociologie et sociétés, 36(1), 229-245. https://doi.org/10.7202/009590ar

\section{Résumé de l'article}

L'auteur analyse le mouvement zapatiste au Chiapas afin de connaître les ajustements cognitifs au cours du cycle de mobilisation qui a précédé le soulèvement du $\mathrm{I}^{\mathrm{er}}$ janvier 1994. D'abord, il souligne le rôle de facteurs classiques mis en évidence par la perspective de la mobilisation des ressources : la structure d'occasions politiques et l'organisation. Ces facteurs ont contribué au développement d'une structure de mobilisation bien spécifique dans la Selva, une région reconnue pour être le bastion des zapatistes. Enfin, l'analyse de témoignages d'Indiens zapatistes révèle la présence de deux processus sociopsychologiques au sein du mouvement, et ce avant la rébellion : la revalorisation du soi collectif et l'amplification des stéréotypes à l'égard des ladinos (non-Indiens). L’Église catholique a contribué à revaloriser le soi collectif. L'amplification des stéréotypes est attribuée à l'influence des guérilleros des Fuerzas de Liberación Nacional (FLN) et, indirectement, à celle de l'autoritarisme de l'État. 


\title{
La rébellion des sans-visage
}

\author{
Analyse de l'émergence du mouvement \\ zapatiste au Chiapas (Mexique)
}

\section{GILLES CORMIER ${ }^{\top}$}

122, rue Turgeon

Montréal (Québec), Canada $\mathrm{H}_{4} \mathrm{C}_{2} \mathrm{~N}_{1}$

Courriel : gilcor@sympatico.ca

$\mathrm{L}$

E $1^{\text {er }}$ JANVIER 1994, des milliers de paysans indiens, la plupart étant armés, envahirent les principales villes du centre de l'un des États les plus pauvres du Mexique, le Chiapas. Le jour même de l'entrée en vigueur de l'Accord de libre-échange nordaméricain (ALENA) entre le Canada, les États-Unis et le Mexique, ce soulèvement fit connaitre au monde entier une région déshéritée, ainsi qu'une organisation qui deviendra un symbole de résistance à la mondialisation, l'Ejercito Zapatista de Liberación Nacional (EZLN). Pour le président Salinas, l'origine de la rébellion était imputable à un groupe d'étrangers extrémistes manipulant les Indiens. Cette explication légitima l'intervention militaire qui suivit. Plusieurs livres ont été publiés sur le mouvement zapatiste. Certains auteurs pensent qu'il s'agit bien d'un mouvement indien (Harvey, 2000; Womack, 1999; Le Bot, 1997). À l'opposé, d'autres auteurs pensent plutôt que le mouvement zapatiste serait contrôlé par les guérilleros et que leur chef serait le dénommé sous-commandant Marcos (Tello Díaz, 2000; De la Grange et Rico, 1998). Selon ces derniers, l'implication de l'évêque de San Cristóbal, Samuel Ruiz, dans le soulèvement armé ne fait aucun doute. La principale lacune de la plupart des interprétations est qu'aucun auteur n'a mené l'analyse du mouvement zapatiste jusqu'au niveau

1. L'auteur est sociologue. Dans le cadre de ses études doctorales, il a séjourné au Chiapas et a effectué des entretiens avec des participants au mouvement zapatiste. 
de la micro-mobilisation en scrutant le cheminement et les témoignages de participants. Nous allons corriger cette lacune en tentant de cerner les ajustements cognitifs chez certains participants au cours du cycle de mobilisation — entre 1983 et 1994 - qui a précédé le soulèvement. Auparavant, nous verrons que deux facteurs classiques, la structure d'occasions politiques et l'organisation, mis en évidence par la perspective de la mobilisation des ressources, ont favorisé le développement d'une structure de mobilisation bien spécifique au Chiapas.

Le Mexique se libère progressivement de l'emprise d'un régime autoritaire dans lequel un seul parti, le Partido Revolucionario Institucional (PRI) s'est maintenu au pouvoir jusqu'à tout récemment, soit pendant soixante et onze ans. Même si ce régime confère beaucoup de pouvoir au président, ce dernier doit composer avec des pouvoirs périphériques, surtout celui des caciques. Au Chiapas, l'État-PRI, avec l'aide de caciques, tenta par tous les moyens de maintenir son influence politique en s'appuyant sur le clientélisme et la corruption. Dans les années 1970, une certaine libéralisation politique favorisa l'apparition d'organisations indépendantes dans trois régions $\mathrm{du}$ Chiapas. Mais une répression intense conduira finalement à l'émergence de l'EzLN au milieu des années 1980. Paradoxalement, la négociation de l'Alena favorisa indirectement le soulèvement du $1^{\text {er }}$ janvier 1994. L'armée mexicaine avait découvert par hasard les insurgés l'année précédente, et de surcroît, elle s'apprêtait à mettre un terme à leurs activités. Pour ne pas nuire à la signature imminente de l'Alena, le président Salinas demanda finalement à l'armée de battre en retraite.

\section{L'APPORT DES THÉORIES CLASSIQUES}

Certains considèrent le mouvement zapatiste comme étant précurseur : «la première révolution du XxI ${ }^{\mathrm{e}}$ (González Casanova, 1996) ou «la première protestation contre la globalisation» (Ramonet, 2001). Le mouvement zapatiste ébranla le système politique mexicain grâce, entre autres, à l'arme idéologique qu'il a su manier avec brio en mettant à contribution les nouvelles technologies de communication comme l'Internet. Certes nous verrons qu'effectivement le mouvement nous oblige à innover dans l'analyse. Mais certaines propositions des théories classiques se vérifient au Chiapas, et ce même si ces dernières ont d'abord été conçues pour analyser les mouvements sociaux dans les sociétés avancées. Nous pensons évidemment aux propositions issues de la perspective de la mobilisation des ressources concernant le rôle des structures d'occasions politiques et de l'organisation dans un processus de mobilisation politique. Cette perspective a remarquablement mis en valeur ces deux dimensions ${ }^{2}$.

L'apport décisif de la perspective de la mobilisation des ressources se résume finalement à deux propositions fondamentales. Ces deux propositions articulent l'action collective avec l'action politique. La première proposition concerne le domaine de l'action politique : un mouvement social émerge en réponse à une structure d'occasions politiques. Celle-ci comporte plus de chances que de menaces politiques. Le terme «structure» signifie que

2. Quelques-uns des principaux représentants de cette perspective sont Oberschall (1973), McCarthy et Zald (1977), ainsi que Tilly (1978). 
ces éléments de l'environnement politique ont un caractère cohérent perçu par des acteurs potentiels. Cette structure est interprétée par des exclus comme étant une possibilité de pénétrer enfin la sphère d'influence politique réservée jusque-là seulement aux membres. Cette première proposition sur la structure d'occasions politiques est liée à une deuxième concernant le domaine spécifique de l'action collective : la mobilisation repose sur une organisation interne vigoureuse. Pour tirer profit des occasions politiques et passer à l'action, un groupe doit disposer d'une organisation interne assez vigoureuse. Son action collective pourra à la rigueur générer de nouvelles occasions politiques.

En mettant en évidence le rôle de ces deux dimensions, cela a permis d'affranchir la perspective de la mobilisation des ressources des conceptions précédentes de la mobilisation axées sur le comportement collectif et notamment sur la frustration relative. Cette dernière conception insistait sur les ruptures au sein du système social engendrant de la frustration relative qui pouvaient ensuite déboucher sur une mobilisation politique. Dans cette conception de la frustration relative, la mobilisation est surtout le résultat de causes internes, voire irrationnelles, impliquant un décalage chez les individus entre leurs attentes et la réalité. En se dégageant des conceptions précédentes, la perspective de la mobilisation des ressources insiste surtout sur les facteurs externes de la mobilisation : les structures d'occasions politiques et l'organisation des ressources. Mais la mobilisation des ressources continue d'impliquer des facteurs internes - psychosociologique, cognitif et culturel — que cette perspective ne pourra pas laisser de côté. À tout le moins, de tels facteurs sont impliqués dans les rapports déjà donnés, ou à activer, entre organisateurs et participants, au sein d'un mouvement social. La perspective de la mobilisation des ressources a tenté de solutionner ce problème qui continue à hanter les sociologues.

À travers l'articulation de ces deux dimensions, la perspective de la mobilisation des ressources a intégré les facteurs internes. Prenons un seul exemple. Le modèle — ou plutôt les deux modèles : celui de la politie et celui de la mobilisation — de Charles Tilly (1978) approfondit ces deux dimensions et leur articulation réciproques. Dans la dimension organisationnelle, il tente de faire davantage place aux facteurs internes sans recourir aux ressources symboliques comme les valeurs partagées. Tilly précise davantage ce qu'est un groupe organisé : "plus le sentiment de l'identité de groupe est fort et davantage est intense le réseau de relations internes, plus le groupe se trouve organisé». Dans le modèle de mobilisation de Tilly, ceux qui s'engagent dans un mouvement social, ce sont les individus les mieux intégrés à leur collectivité. De surcroît, ils le font pour défendre des intérêts collectifs. Le modèle de politie concerne les relations entre les groupes au sein d'une société selon leur position par rapport à la sphère d'influence politique. Dans ce dernier cas, il s'agit des chances ou menaces politiques. La répression, surtout de la part du gouvernement, affecte la capacité d'un groupe contestataire d'entreprendre une action collective en la rendant plus coûteuse. Ces deux dimensions agissent l'une sur l'autre. Cela explique d'ailleurs l'apparente contradiction entre les propositions de la perspective de la mobilisation des ressources. Si la mobilisation est le fait de groupes exclus du système d'influence politique, ceux-ci doivent néanmoins avoir un certain degré d'intégration dans la société. Ils doivent de plus avoir des 
organisations, des professionnels et des ressources pour profiter des occasions politiques offertes pour agir collectivement.

Plus on tente d'améliorer l'articulation entre ces deux dimensions, plus on voit se dessiner deux processus distincts : la mobilisation des individus par l'entremise de leur forte intégration et identification à un groupe — donc impliquant les dimensions sociopsychologique, culturelle et cognitive — et l'action politique stratégique étant surtout le fait des organisations. Dans le cas du mouvement zapatiste, les communautés actives sont certes isolées de la sphère d'influence politique, mais entretiennent des réseaux complexes de relations entre elles, ainsi qu'avec des organisations nationales, voire internationales. L'analyse de ces réseaux — qui évoluent constamment — nous permettrait d'approfondir la compréhension de l'articulation des dimensions fondamentales de la mobilisation. Pour ce faire, il faut notamment dégager les cadres interprétatifs des participants à un mouvement social (Chazel, 1997; Snow et al., 1986). Nous allons nous engager dans cette avenue. Mais d'abord voyons comment on peut appliquer les concepts fondamentaux de la perspective de la mobilisation des ressources à l'analyse du mouvement zapatiste.

\section{STRUCTURE D'OCCASIONS POLITIQUES ET ORGANISATION}

La structure d'occasions politiques est un des concepts fondamentaux de la perspective de la mobilisation des ressources (McAdam, 1982; Tarrow, 1998). Tarrow la définit à partir des quatre éléments suivants : l'ouverture à la participation, la division au sein de l'élite, la présence d'alliés et le changement de dirigeants. Le terme de «structure» n'est peut-être pas le meilleur. La structure d'occasions politiques a été confondue avec les nouvelles occasions offertes par un État-providence. Le concept désigne un phénomène plus large : une situation certes changeante, mais cohérente aux yeux des acteurs potentiels. Cela rend finalement possible une action collective structurée menée par une organisation. Cette définition du concept de structure d'occasions politiques, tout comme celle d'ailleurs d'organisation de l'action collective, impliquent finalement des facteurs subjectifs - l'interprétation d'une situation donnée par des acteurs potentiels — dont il faut tenir compte.

Selon la perspective de la mobilisation des ressources, il s'agit d'observer, en période de croissance économique, la mobilisation de certains groupes sociaux qui avaient été jusque-là exclus de la sphère d'influence politique. Les États-Unis et l'Europe des années 1960 et 1970 offrent d'ailleurs un terrain privilégié pour observer l'apparition de nouveaux mouvements sociaux. Les guerres, l'industrialisation et la croissance démographique sont quelques exemples de facteurs qui agissent sur la structure d'occasions politiques et sur l'organisation interne d'un groupe. Selon McAdam (1982), les chances politiques s'élargissent avec le temps. Elles atteignent un sommet, puis déclinent. Elles seront d'autant mieux saisies que l'organisation interne d'un groupe sera vigoureuse et qu'au même moment la situation sera interprétée différemment. Bref, la mobilisation politique n'est pas à la portée d'un groupe en situation extrême de pauvreté et d'isolement. Pour Tarrow (1998), l'évolution n'est pas aussi linéaire. D’ailleurs, la structure des chances politiques 
varie autant dans l'espace que dans le temps. Un même État peut à un moment donné offrir des chances politiques à certains acteurs tout en exerçant une forte répression à l'égard d'autres acteurs. Une structure d'occasions politiques possède néanmoins un cycle qui s'ouvre avec l'action collective de nouveaux acteurs. Leur action collective peut ouvrir de nouvelles occasions politiques autant à eux-mêmes qu'à d'autres acteurs. L'État va réagir devant ces nouveaux mouvements sociaux. Il va tenter par différents moyens, réformes ou répression, de leur mettre un terme.

Dans un régime autoritaire, comme au Mexique, la structure des chances politiques est bien différente de ce qu'on retrouve en Europe ou aux États-Unis à la même époque $e^{3}$. Une structure d'occasions politiques est néanmoins apparue au Chiapas dans les années 1970. Elle reposait principalement sur deux acteurs : l'État et l'Église catholique. L'action de l'État-PRI fut ambiguë. Il offrit une certaine libéralisation politique (Couffignal, 1988), mais en même temps un décret présidentiel, qui créa la réserve Lacandona, affecta une trentaine de communautés indiennes de la Selva. L’Église catholique fut un allié des Indiens. Elle intervint sur deux plans : par son action pastorale auprès des communautés indiennes et par son engagement dans l'organisation du Congrès indigène du Chiapas en 1974. Cela met en évidence le caractère international de cette occasion politique. L'Église catholique avait procédé à une vaste réforme à la suite du deuxième concile du Vatican entre 1962 et 1965. L'épiscopat latino-américain avait fait sien le principe d'adaptation de la foi à la culture des peuples lors de sa conférence à Medellín en 1968. Enfin, l'évêque de San Cristóbal de las Casas, Samuel Ruiz, appliqua ce principe à sa pastorale auprès des communautés indiennes. Concernant cette pastorale indienne, on doit analyser ses caractéristiques bien spécifiques plutôt que de la qualifier simplement de «théologie de la libération» (Meyer, 2000).

Concernant le rôle de l'organisation, à la faveur d'une structure d'occasions politiques, de nouvelles ressources à la disposition d'un groupe défavorisé peuvent être mobilisées dans la défense de ses intérêts. Pour ce faire, la présence d'une structure de mobilisation (Tarrow, 1998), où l'organisation formelle et des professionnels jouent un rôle important, permet de mobiliser ces nouvelles ressources pour entreprendre des actions collectives. En 1974, le Congrès indigène du Chiapas fut une véritable chance politique pour les Indiens. Cet événement fut organisé par l'évêque de San Cristóbal à la demande du gouverneur du Chiapas. À la suite du Congrès indigène, de nouvelles organisations surgirent dans trois régions : la dépression centrale, l'Altos et la Selva. Des promoteurs d'organisations nationales aidèrent à la formation de nouveaux leaders indiens politisés dans ces trois régions. Les structures de mobilisation mises en place varièrent d'une région à l'autre. Dans la région centre, le leadership des organisateurs est fort. Dans la région Altos, l'organisation a développé des liens avec un parti d'opposition, le Partido de la Revolución Democrática (PRD). Dans la Selva, les communautés mobilisées maintiennent leur autonomie.

3. Contrairement au mouvement noir américain, le mouvement indien au Chiapas n'a pu amener l'État fédéral à intervenir en sa faveur. 


\section{LA DIMENSION SOCIOPSYCHOLOGIQUE}

Il n'existe pas encore de théorie définie sur le rôle de la dimension sociopsychologique et cognitive dans la mobilisation politique. Nous allons établir quelques balises. Sur quels cadres les ajustements se sont-ils effectués chez les participants? La condition de peones $^{4}$ n'existe plus dans les années 1970, mais le souvenir demeure notamment chez les anciens. Nous allons donc évaluer l'impact du clivage entre peones et ladinos sur les ajustements cognitifs des Indiens zapatistes. Enfin, nous disposons tout au plus de quelques pistes théoriques : les modèles de l'identité sociale (Tajfel et Turner, 1979; Hogg et Abrams, 1988) et de l'imputation de causalité (Ferree et Miller, 1985; Pettigrew, 1979). Les théories de l'identité sociale supposent chez l'individu un processus d'identification à deux dimensions, individuelle et collective, selon son statut et son pouvoir dans la société. Selon Tajfel et Turner, les individus ont surtout recours à des cadres interprétatifs individualistes en l'absence d'une «alternative cognitive». Cette dernière consiste en la perception d'un changement social dans leur situation par les participants éventuels à une mobilisation politique. Ce concept ne permet pas une analyse minutieuse des ajustements cognitifs chez les participants à un mouvement spécifique. Toutefois, cette théorie nous amène à questionner la disponibilité des cadres individualistes dans un contexte culturel collectiviste comme celui du Chiapas ${ }^{5}$. Dans un tel contexte, nous supposerons qu'une dimension de la personnalité est prédominante : le moi collectif (Triandis, 1989).

Nous avons développé une définition plus élaborée du moi collectif que celle proposée par Triandis afin de tenir compte des possibilités d'ajustements cognitifs dans un processus de mobilisation politique spécifique. Nous parlerons alors de soi collectif ${ }^{6}$ qui permettrait à un individu d'intégrer divers mois collectifs, de reconnaître que certains mois collectifs sont rattachés à des groupes distincts et éventuellement de respecter ces derniers. En effet, d'une expression prototypique ou traditionnelle de la communauté indienne, les Indiens du Chiapas vont découvrir peu à peu diverses formes d'expression

4. Ouvrier semi-esclave dans une grande exploitation agricole.

5. Par «collectiviste», on entend le fait que des individus soient fortement attachés à leur communauté et qu'ils subordonnent leurs buts personnels à des buts collectifs.

6. Le soi collectif rend compte d'un phénomène sociopsychologique spécifique. Il concerne exclusivement les représentations de soi en tant que membre d'une organisation, d'un groupe ou d'une collectivité. Le soi collectif est un ensemble de représentations qui se rapportent à soi-même et aux autres, mais sous l'angle spécifique de l'interaction entre membres d'organisations, de groupes ou de collectivités. Ces interactions prennent en réalité diverses formes — allant du mépris jusqu’à la reconnaissance — qui influencent la composition du soi collectif des individus. La composition du soi collectif suppose chez l'individu une capacité d'objectiver son appartenance à un groupe : la conscience de soi collectif. Celle-ci est capable de dépasser ses propres limites et de s'ouvrir sur le monde en intégrant divers mois collectifs. Cela exige cependant des efforts de reconnaissance mutuelle prenant appui sur la réalité, c'est-à-dire la reconnaissance de communautés réelles. Un soi collectif nouvellement recomposé peut s'appuyer sur la constitution d'une communauté élargie, voire idéale, mais incluant des communautés existantes. L'une des particularités des Indiens zapatistes est justement d'avoir maintenu ce lien avec des communautés existantes. Même s'ils n'ont pas été exempts d'amplification des stéréotypes à l'égard des ladinos, ils ont évité une suridéalisation de leur moi collectif. On retrouve une telle suridéalisation chez certains islamistes radicaux, cf. le témoignage d'un «fou d'Allah» de Khaled Al-Berry (2002). 
de soi collectif. Par exemple, concernant leur rapport à l'État, diverses formes d'engagement apparaîtront dans la Selva à partir des années 1980. Il y aura les communautés priistes — liées à l'État-PRI —, les indépendantes — ces dernières se subdiviseront entre progouvernementales et les autres - et enfin les zapatistes qui, elles, seront en faveur de la lutte armée pour faire avancer leurs revendications. Mais au départ, la plupart des communautés cherchèrent à faire reconnaître par l'État de nouveaux rapports plus égalitaires.

Un exemple concret nous permettra de constater la prédominance du moi collectif. Le 7 juin 1996, deux prisonniers zapatistes, qui avaient été accusés de «terrorisme, rébellion et conspiration », sont finalement acquittés et libérés au Chiapas. À sa sortie de prison, l'un d'eux, Sebastián Entzin Gómez - le seul Indien, l'autre étant un journaliste métis —, déclara à la presse qu'il retournerait à sa communauté demander pardon :

J'ai mal fait mon travail. J'ai désobéi aux ordres et c'est pour ça que j'ai été fait prisonnier. Je suis disposé à accepter n'importe quelle punition dans ma communauté pour l'erreur que j'ai commise, bien que ce ne soit pas quelque chose de mal puisque je n'ai pas volé et rien de tel sinon qu'ils m’ont attrapé (La Jornada, 8 juin 1996, p. 8).

Selon ce que nous avons observé sur le terrain, le moi collectif est la dimension prédominante de la personnalité des Indiens du Chiapas. Il y a d'ailleurs deux processus fondamentaux : la décomposition et la recomposition du soi collectif. Cette recomposition peut engendrer des processus de revalorisation du soi collectif et d'amplification des stéréotypes sur lesquels nous insisterons. Le soi collectif traditionnel des Indiens du Chiapas est en décomposition. Il est lié à la survivance de la communauté traditionnelle — soutenue d'ailleurs par l'État-PRI — dans la région d'Altos. Ce moi collectif a de plus en plus de mal à se maintenir à travers les bouleversements sociaux et politiques, mais sa décomposition ne se fait pas sans heurt. Elle se manifeste, entre autres, par des actes d'une violence inouïe dans cette région comme ce massacre par un groupe paramilitaire de 45 villageois, en majorité des femmes et des enfants, à Acteal le 22 décembre $1997^{7}$. Pour endiguer cette décomposition, les solutions de rechange individualistes sont rarissimes ${ }^{8}$.

En ce qui a trait à l'interaction entre individus appartenant à des groupes distincts, certaines théories de l'imputation de causalité insistent alors sur le rôle des stéréotypes.

7. On trouve une explication intéressante de ce conflit par Shannan L. Mattiace (2001). Selon l'auteur, la petite communauté de Santa Marta dans le municipio de Chenalhó — où ont été recrutés les paramilitaires aurait été négligée autant par l'EZLN que par le gouvernement du Chiapas dans leurs efforts pour aménager de nouveaux municipios.

8. Une conjoncture économique favorable, notamment grâce aux investissements publics dans les infrastructures et l'exploitation pétrolière au cours des années 1960 et 1970 a permis d'améliorer la condition de certains Indiens, devenus journaliers ou entrepreneurs, et de développer progressivement chez eux des cadres interprétatifs relevant de la dimension individuelle du moi. Des occasions du même genre ont aussi été offertes par l'État-PRI à travers les relations clientélistes tissées avec des caciques indiens. Certains individus quittent encore aujourd'hui leur communauté pour un séjour prolongé afin de tenter leur chance ou aller chercher une formation dans un centre urbain comme San Cristóbal de las Casas. Bref, le moi individuel est bien présent chez certains, appartenant à un bassin de leaders potentiels courtisés par les organisations externes, même si l'autre dimension continue à prédominer. 
Selon certaines études, la perception chez les membres d'une organisation clandestine armée amplifie les stéréotypes à l'égard de l'adversaire (della Porta, 1992). Ce dernier devient alors un ennemi auquel est dénié toute humanité. Cette perception des groupes en conflit rend quasiment impossible la réconciliation des parties. Elle serait même imperméable à tout changement de stéréotypes. Par ailleurs, la théorie de Hewstone (1989) prédit que, dans un conflit intense, une réduction des tensions est possible lorsqu'il y a un changement de stéréotypes à l'égard de l'adversaire. Ces modèles sont intéressants pour diverses raisons. Il semble en effet que le phénomène de "stéréotypage» soit présent dans un contexte culturel collectiviste comme celui du Chiapas et, de surcroît, où subsiste toujours des traces d'un clivage ancestral. Tout en admettant qu'un changement de stéréotypes entre groupes adverses va atténuer l'hostilité entre eux, l'analyse se complique lorsqu'on tient compte des éléments politiques fondamentaux : la présence des autres groupes, alliés ou non, ainsi que le caractère politique du conflit qui se définit, entre autres, à travers les rapports des groupes avec l'État. Au Chiapas, l'Église aurait contribué à la revalorisation du soi collectif chez les Indiens, mais qu'en est-il de leur perception à l'égard des ladinos? Les Indiens ont-ils considéré les missionnaires blancs qui ont valorisé la communauté indienne comme ayant un comportement atypique par rapport à celui de la plupart des ladinos? Par la suite, le retrait de l'Église des projets sociocommunautaires ainsi que l'autoritarisme de l'État auraientils facilité la tâche aux guérilleros pour amplifier des stéréotypes négatifs à l'égard des ladinos, voire jusqu'à justifier la lutte armée contre les ladinos? Pour répondre à ces questions, nous avons retenu une hypothèse. Au moment de son émergence, le mouvement zapatiste aurait été la résultante de deux processus sociopsychologiques au sein d'une même structure de mobilisation : la revalorisation du soi collectif et l'amplification des stéréotypes à l'égard des ladinos.

Pour approfondir le rôle de la dimension sociopsychologique, nous avons privilégié l'analyse de la structure spécifique de mobilisation mise en place dans le Selva. Ce fut surtout dans la Selva que l'action pastorale de l'Église eut le plus d'impact. Son action pastorale, combinée à un processus de colonisation ayant une signification particulière pour les Indiens - un recommencement —, favorisa le rétablissement de la communauté indienne. Les Indiens s'inspirèrent du comón — les membres d'une communauté qui, réunis en plénière, dictent les règles à suivre - et de la réactualisation de leur méthode de discussion en assemblée, l'acuerdo, c'est-à-dire qu'au milieu des murmures des membres se font entendre afin de parvenir à un consensus sans avoir recours au vote. De nouvelles communautés, plus «modernes» (Krauze, 1999), apparurent. En 1974, au cours du Congrès indigène, on utilisa pour la première fois leur méthode de discussion en dehors de son cadre communautaire. Avec l'aide d'organisateurs externes, cette méthode fut mise à contribution dans la formation d'une des plus grandes organisations du Chiapas, la Unión de Uniones. Ce cheminement favorisa la revalorisation d'eux-mêmes. En même temps, la perception à l'égard des ladinos était appelée à subir des ajustements. Le stéréotype du finquero ladino rico, le fermier blanc riche, hérité du

9. Cette expression désigne les non-Indiens que ceux-ci soient blancs ou métis. 
clivage passé, était toujours présent dans l'esprit des Indiens, surtout chez les anciens, mais sans pouvoir pour autant justifier la lutte armée. Ce stéréotype ne correspondait plus au monde ladino devenu plus complexe et avec lequel les Indiens souhaitaient maintenant développer des rapports nouveaux et égalitaires. Concernant leur tentative d'établir de nouveaux rapports avec l'État au cours des années 1970, le témoignage d'un colon de la Selva est éloquent :

Bien que nous ayons tenté d'établir une relation de dialogue avec les gouvernements, cela ne fut pas possible. Pour eux, il y avait deux seuls types d'organisation, nous étions blancs ou noirs : ou tu étais avec eux ou tu étais contre eux. Bien que nous ayons essayé de leur dire que nous n'étions ni l'un ni l'autre, que nous voulions une nouvelle relation, ils ne l'acceptèrent pas (Legorreta Díaz, 1998, p. 93-94).

Finalement, la Selva deviendra le bastion de l'Ezln. Mais les organisations indiennes de cette région seront alors divisées.

\section{LE CLIVAGE ANCESTRAL}

Il s'agit maintenant d'évaluer si ce grand clivage entre Indiens et ladinos a pu donner lieu à une perception du conflit qui aurait justifié la lutte armée. Selon María del Carmen Legorreta Díaz (1998), l'Église aurait contribué largement à développer, chez les Indiens de la Selva, une «utopie de la libération». Cette idéologie était, selon elle, une sorte de christianisme mâtiné de marxisme et d'indianisme millénariste. L'action des agents de pastorale aurait été ambiguë. Si l'idéologie qu'ils ont diffusée a eu pour effet d'amener les Indiens à accepter la lutte armée, elle reconnaît cependant que la pastorale a aussi contribué à développer une meilleure estime d'eux-mêmes. Son interprétation du rôle du diocèse est donc mitigée. Notre interprétation diverge. À notre avis, la pastorale indienne aurait plutôt eu pour effet de revaloriser leur soi collectif à travers une forme de reconnaissance mutuelle. Cela aurait atténué l'amplification de stéréotypes et fait en sorte que les affrontements armés ne durent qu'une douzaine de jours. Après le soulèvement, les zapatistes ont accepté, sous la pression de la société civile, de dialoguer avec le gouvernement mexicain. Cela est en partie attribuable à l'expérience particulière — notamment l'interaction avec les métis — des Indiens de la Selva. Avec certains représentants ladinos, qu'ils aient appartenu à l'Église catholique ou à une organisation nationale, les Indiens ont développé une relation différente au fil des ans. Ce développement du processus sociopsychologique endogène sera précieux : la reconnaissance des autres a acquis alors une place importante.

Ce processus de revalorisation du soi collectif à travers la reconnaissance mutuelle est relativement récent chez les Indiens. Un autre type de perception, voire d'idéologisation du conflit n'est peut-être pas écartée. Par exemple, un indianisme plus radical peut-il émerger? Ce clivage ancestral entre Indiens et ladinos engendra quelques soulèvements indiens dont cette légendaire «guerre de castes» redoutée par les ladinos qui survint en 1869. La répression qui suivit marqua le début de l'exploitation à plus grande échelle des Indiens. À la fin du XIx ${ }^{\mathrm{e}}$ siècle, le nombre de fincas augmenta de façon phénoménale au Chiapas, entre autres, grâce aux capitaux étrangers. La vie des Indiens en 
fut profondément transformée. Leur destinée était dorénavant liée à celle de la finca. Dans la région d'Altos, les communautés indiennes traditionnelles, les lums, permirent aux Indiens de résister en réactualisant des croyances et des rites ancestraux que l'action des missionnaires avait jadis inhibés. Au cours de son règne, qui dura soixante et onze ans, l'État-PRI réussit à étendre son influence jusque dans ces communautés indiennes traditionnelles. Après la présidence de Cardénas, à la fin des années 1930, au cours de laquelle ce dernier procéda à une vaste redistribution de terres, le PRI réussit à s'implanter à l'intérieur des communautés indiennes (Rus, 1995). Des chefs corrompus, des caciques, furent cooptés grâce au clientélisme et à la corruption. Au début des années 1970, la condition de peones avait disparue. Les Indiens ont alors entamé un processus d'appropriation du soi collectif ${ }^{10}$.

Nous disposons d'un témoignage récent d'un Indien tzotzil — qui ne se définit pas comme étant zapatiste - de la région Altos du Chiapas. En fait, dans son texte, Jacinto Arias nous livre sa vision de l'histoire des Indiens du Chiapas. On découvre comment il perçoit leur cheminement pour retrouver leur dignité; bref, la revalorisation de leur soi collectif. En simplifiant, nous pouvons résumer son propos comme ceci : avant la conquête, les Indiens avaient une vision de l'univers comme étant bien ordonné et dominé par les maîtres de la vie. Avec l'influence du catholicisme, ceux-ci cédèrent la première place au «Seigneur», El ajvalil. Le terme de «seigneur» dans le sens de «propriétaire» ne faisait pas partie de leur vision ancestrale. Après la conquête, ce terme servit à qualifier l'attitude des chefs blancs et métis à l'égard de la terre et des Indiens qui l'habitaient. Cela suscita dès le début de la méfiance, mais depuis peu on a commencé un "combat pour s'appartenir à soi-même» à travers la récupération des us et coutumes, ainsi que du gouvernement. Ce témoignage révèle une caractéristique idéologique qui anime les Indiens du Chiapas, en particulier dans la région Altos ${ }^{11}$. Ces derniers tiennent à affirmer clairement leur différence d'avec les ladinos. On retrouve cette même caractéristique dans certains témoignages du commandant David, un leader indien zapatiste de cette région.

\section{L'ÉMERGENCE DE L'EZLN}

On situe les débuts clandestins de l'EzLn au mois de novembre 1983. À cette époque, la conjoncture politique locale était défavorable aux organisations indépendantes démocratiques au Chiapas. Cette situation favorisa d'une certaine manière l'implantation d'une organisation nationale clandestine - quelques guérilleros des Fuerzas de Leberación Nacional (FLN) - ainsi que la radicalisation de certains Indiens qui participaient déjà aux actions collectives des organisations indépendantes démocratiques. Malgré le caractère répressif de l'État à l'échelle locale, cela n’a pas engendré automatiquement une réaction violente. L'analyse d'une rébellion doit tenir compte autant de la structure de mobilisation des protagonistes que de leurs processus sociopsychologiques. Le processus

10. Un texte remarquable, notamment du point de vue de la réflexion actuelle d'un Indien du Chiapas, sur leur identité collective, est sans aucun doute celui de Jacinto Arias (1994).

11. Les Indiens de cette région ont chassé la plupart des métis qui y résidaient. 
sociopsychologique de recomposition du soi collectif — dont nous avons supposé l'apparition au cours d'un premier cycle de mobilisation dans les années 1970 — se serait maintenu au cours du cycle de protestation, considéré plus radical, qui a suivi au cours des années 1980. Dans l'entretien que nous avons effectué en mai 1996 avec un zapatiste civil d'une communauté indienne la Selva, appartenant à l'ethnie tojolabale, ce dernier témoigne par ses propos de la présence de ce processus sociopsychologique. Ce processus s'appuie sur une expérience organisationnelle régionale et autonomiste ${ }^{12}$. À une question sur les changements dans sa communauté depuis que celle-ci a adhéré au mouvement zapatiste, voici sa réponse :

Cela n'a rien changé dans les collectivités indiennes, c'est-à-dire dans la situation de vie, parce que nous n'avons rien reçu. Nous avons survécu grâce à la société civile qui nous a aidés. Mais nous n'avons pas pu semer, ni récolter. Et c'est là qu'on nous a aidés, avec l'alimentation. C'est ainsi que nous avons survécu. Dans ce sens, les collectivités n’ont pas changé ou n'ont pas vécu d'amélioration. Mais dans un autre sens, elles ont changé. Et cela servit d'expérience à d'autres communautés, à d'autres collectivités. Dans le sens de l'unité, de prendre soin de nous-mêmes, de ne pas nous vendre au gouvernement, d'avoir confiance en nous-mêmes, les pauvres, et de ne pas croire à l'idéologie du gouvernement qui vient faire beaucoup de promesses. Il répète la même chose que disent les partis et offrent beaucoup de choses avant de prendre le pouvoir. Lorsqu'ils l'ont pris, ils oublient ceux qui ont voté pour eux et qui les ont portés au pouvoir. En ce sens, nous avons changé. Nous avons compris (Cormier, 2002, p. 200).

Ce processus aurait atténué les effets de l'amplification des stéréotypes à l'égard de l'adversaire. De plus, le processus de revalorisation du soi collectif serait assez répandu dans la Selva et irait bien au-delà de l'organisation formelle du mouvement zapatiste. Ce processus repose essentiellement sur une identité communautaire forte dans certaines sous-régions de la Selva (Leyva Solano et Ascencio Franco, 1996) ainsi que sur l'expérience d'organisation régionale autonome. Celle-ci, renforcée par des réseaux de solidarité avec diverses organisations qui ont pris le relais de l'Église catholique, débouchera finalement sur la création de municipios $^{13}$ autonomes, mais qui seront ensuite démantelés par l'État. Le témoignage ci-dessus exprime une méfiance à l'égard de la classe politique mexicaine. Cette perception influence leur organisation intercommunautaire. Mais les Indiens ont réussi à faire la part des choses, bien que certains aient à un moment donné amplifié les stéréotypes à l'égard des ladinos. Seule une minorité de ladinos, voire même d'Indiens, qui détiennent un certain pouvoir, s'oppose farouchement à l'autonomie indienne, notamment à cause de la menace qu'elle représente pour ses intérêts ${ }^{14}$. Après leur rébellion, les Indiens zapatistes découvrirent qu'ils partageaient avec beaucoup de Mexicains cette même méfiance à l'égard de la classe politique.

12. Pour une analyse de l'organisation régionale autonome des Indiens tojolabales du sud-est du Chiapas, voir l'article de Shannan L. Mattiace (2001).

13. Entité administrative équivalente à une commune ou une municipalité.

14. Par exemple, l'organisation intercommunautaire des Indiens tojolabales a débuté avec la prise en charge du transport local à cause des abus commis par une entreprise appartenant à un cacique ladino. Ce processus a donc frappé de plein fouet les intérêts économiques de cette entreprise ladina. 
Au début des années 1980, la crise économique mondiale eut des répercussions au Chiapas. La fin du premier cycle de protestation s'annonçait alors. La stratégie de l'ÉtatPRI fut autoritaire à l'égard des organisations indiennes indépendantes du Chiapas. Elle se résumait à diviser, coopter et réprimer. Cette stratégie a mis beaucoup de temps à donner des résultats dans la Selva. À la fin des années 1980, l'ARIC — regroupement d'ejidos ${ }^{15}$ dans la Selva — va finalement négocier avec l'État un règlement aux problèmes agraires. L'État-PRI réussit ainsi à avoir une certaine influence sur cette région, mais trop tard. Les organisations indépendantes étaient déjà divisées. La répression du mouvement paysan était brutale au milieu des années 1980. On dénombrait deux assassinats de leaders paysans en moyenne par mois au Chiapas ${ }^{16}$. Un deuxième cycle de protestation s'était déjà amorcé dans lequel une organisation nationale clandestine, les Fuerzas de Liberación Nacional (FLN), prit le leadership. Selon notre hypothèse, les guérilleros vont réussir, avec l'aide indirecte de l'autoritarisme de l'État, à amplifier les stéréotypes à l'égard des ladinos chez certains Indiens de la Selva.

\section{L'AMPLIFICATION DES STÉRÉOTYPES}

Qu'est-ce qu'on entend par amplification des stéréotypes? Il s'agit ici d'un processus sociopsychologique, sous-jacent à la recomposition du soi collectif, au cours duquel des individus adoptent une vision militariste — de leur conflit avec les ladinos — justifiant la lutte armée. Les guérilleros ont joué un rôle indéniable dans ce genre d'amplification des stéréotypes, mais ils devaient cependant composer avec des communautés indiennes autonomes. Le recrutement des communautés indiennes fut favorisé par la perte de droits agraires avec la modification apportée par le président Salinas à l'article 27 de la Constitution mexicaine en février $1992^{17}$. Certaines communautés optèrent alors pour la lutte armée. Cette époque marqua également un changement dans la méthode de recrutement des FLN-EZLN. Des communautés indiennes entières furent mobilisées par blocs. La formation idéologique des Indiens zapatistes aurait été alors beaucoup moins élaborée, voire inexistante. À partir de l'analyse de témoignages de participants au mouvement zapatiste, nous avons pu constater ces ajustements cognitifs chez les Indiens de la Selva. L'influence de la communauté dans leur perception des groupes en conflit est palpable. Cette perception possède un caractère bien plus politique que militaire. Bref, elle n'a pas la caractéristique d'une amplification telle que l'adversaire serait considéré comme un ennemi dans un conflit militaire. De plus, les communautés mobilisées ont une voix au chapitre dans la structure de fonctionnement interne de l'EzLn. Après le soulèvement,

15. Une partie de terre nationale ou communale réservée de droit à une communauté de paysans. Ces derniers, regroupés en ejidos, ne sont pas propriétaires de la terre, mais disposent de son usufruit.

16. Cf. le rapport d'Amnistie internationale (1986).

17. Avant d'être modifié, l'article 27 de la Constitution permettait aux paysans pauvres de disposer de la terre à travers la propriété sociale. L'État, tout en restant propriétaire de la terre, reconnaissait les communautés paysannes formées en ejidos comme l'unique bénéficiaire de son usufruit. Les terres d'un ejido étaient inaliénables. Certains considéraient ce mécanisme constitutionnel, qui permettait d'ailleurs l'expropriation de la propriété privée, inadapté au contexte de mondialisation économique et comme faisant obstacle à la modernisation de l'économie mexicaine. 
l'importance des communautés indiennes s'est d'ailleurs accrue au sein du mouvement zapatiste, voire à l'échelle nationale à travers le Congrès national indigène.

Dans ce second cycle de protestation, de 1983 à 1994, au cours duquel émergea progressivement l'EzLn, l'influence du processus de revalorisation du soi collectif continua à se faire sentir au sein du mouvement zapatiste, notamment sur le plan de la perception des groupes en conflit. Ce processus de revalorisation du soi collectif a atténué les effets du processus d'amplification. D'autres facteurs, que les guérilleros ont eux-mêmes contribué à produire, ont eu le même effet : l'ajustement des stéréotypes reliés au rôle de la femme indienne dans sa communauté ainsi que le fait de n'effectuer aucune offensive militaire. Malgré tout, les guérilleros, ainsi qu'indirectement l'autoritarisme de l'État, ont contribué à l'amplification des stéréotypes à l'égard des ladinos justifiant ainsi la lutte armée. Mais cette amplification fut de courte durée et s'est atténuée après les affrontements armés de janvier 1994. Finalement, lors du soulèvement, le mouvement zapatiste fut la résultante, au sein d'une même structure de mobilisation politique, de deux types principaux d'ajustements cognitifs : la revalorisation du soi collectif et l'amplification des stéréotypes à l'égard des ladinos.

Pour ce qui est du processus d'amplification des stéréotypes, l'analyse de témoignages de leaders indiens zapatistes nous révèle sa présence. Au tout début du mouvement zapatiste, selon certains témoignages que l'on retrouve dans le livre controversé de Bertrand De la Grange et Maite Rico (1998), les guérilleros auraient formé une «école de cadres». Celle-ci favorisa indéniablement l'amplification des stéréotypes à l'égard des ladinos justifiant la lutte armée, et ce, en particulier chez certains Indiens qui furent parmi les toutes premières recrues. Les auteurs citent un extrait d'un article de Moisés, de la revue clandestine des FLN, Nepantla, publié en 1991.

Pour attirer les masses, écrivait Moisés, il faut les politiser et les conscientiser, leur expliquer qu'il y a des solutions à la misère et qu'elles ne doivent compter sur personne d'autre que sur elles-mêmes pour se libérer...

Une fois, poursuivent les auteurs, qu'il avait réussi à convaincre «le chef du groupe de travail collectif», Moisés était invité à s'adresser aux autres membres du village.

Puisque les riches et le gouvernement ne comprennent pas la voie pacifique, disait Moisés aux membres du village, il faut nous organiser d'une autre manière... Je leur parle, explique Moisés, de la vie dans les pays socialistes, de la lutte au Salvador et au Guatemala. C'est la seule manière de faire entendre raison aux riches. Nous devons donc organiser la lutte armée pour conquérir la liberté et nous défendre (De La Grange et Rico, 1998, p. 99).

Ce texte témoigne d'une amplification des stéréotypes à l'égard de l'adversaire, mais teintée de marxisme-léninisme. À l'instar du major Moisés, dont nous avons analysé en détail quelques-uns des témoignages, et compte tenu de leur expérience réelle de la guerre, certains combattants auront du mal, après le soulèvement, à laisser de côté ce genre de cadres militaristes. En juin 1996, une guérilla traditionnelle, l'Ejército Popular Revolucionario (EPR), est apparue dans l'État de Guerrero au Mexique. En 1996, le sociologue français, Yvon Le Bot (1997) a interviewé le major Moisés. Il lui a demandé s'il était prêt à reprendre la lutte armée, comme venait de le faire récemment l'EPR. 
Dans sa réponse, nous voyons qu'il reste attaché à une certaine vision militariste du conflit :

Ça pourrait bien arriver, parce que le gouvernement démontre à chaque occasion que c'est ce qu'il veut. Nous, on dit qu'on ne veut pas la guerre, mais de l'autre côté il y en a qui la veulent. Ça ne serait pas tellement étonnant, ils nous provoquent sans arrêt pour relancer la guerre.

Mais ce serait tomber dans le piège, s'exclame Le Bot.

Ça dépend de la gravité de la situation et de la décision de nos camarades. C'est surtout ça, si tu ne fais pas ce que décide le peuple, le peuple t'échappe... Ce n'est pas à nous, les militaires, de décider. On fera ce que diront les gens. C'est beau de faire ce que le peuple dit (Le Bot, 1997, p. 280-281).

Cependant, nous ne retrouvons pas un tel attachement aux cadres militaristes dans le témoignage livré à la même époque par le leader indien zapatiste, Tacho. Ce dernier a participé au soulèvement en tant que réserviste. L'amplification des stéréotypes à l'égard de l'adversaire s'est donc manifestée différemment chez les participants selon la catégorie à laquelle ils appartenaient au sein du mouvement zapatiste, voire selon le cheminement de chacun. Elle fut intense chez certains combattants qui furent parmi les premières recrues des FLN, et qui seraient sans doute passés par l' «école de cadres » des FLN. Elle fut moins intense chez les réservistes qui ont participé aux affrontements de janvier 1994. Dans le cas des zapatistes civils dans les communautés de la Selva, cette amplification fut nettement atténuée.

\section{CONCLUSION}

Notre interprétation du mouvement zapatiste met l'accent sur la dimension sociopsychologique ainsi que sur deux facteurs classiques : l'organisation et la structure d'occasions politiques. Elle nous permet de préciser le rôle des principaux protagonistes : des Indiens attachés à leurs communautés qui ont développé des liens serrés entre elles sur le plan régional, ainsi qu'avec des organisations nationales et internationales. Notre analyse apporte une compréhension nouvelle de la mobilisation politique, en particulier dans un contexte culturel spécifique que nous avons qualifié, à défaut d'une meilleure expression, de collectiviste. Des organisations poursuivant des fins radicales en ayant recours à la violence politique sont actives un peu partout dans le monde. Leurs membres sont pour la plupart issus d'un tel contexte culturel. Notre hypothèse est que l'implication des individus dans de telles organisations s'appuie sur les processus sociopsychologiques que nous venons de décrire : la décompositionrecomposition du soi collectif et ses processus sous-jacents, la revalorisation du soi collectif et l'amplification des stéréotypes à l'égard de l'adversaire.

Le soi collectif exerça une influence indéniable dans le mouvement zapatiste. Sa recomposition s'appuya sur une expérience particulière liée à des communautés réelles et permettant une amorce de reconnaissance mutuelle entre Indiens et ladinos. À notre avis, les chances de pacification d'un conflit reposent sur la vigueur de ce processus de 
recomposition du soi collectif à travers la reconnaissance mutuelle (Mead, 1967). Finalement, la première arme, voire la plus efficace, à utiliser contre le terrorisme n'estelle pas la reconnaissance d'une communauté dont les droits élémentaires sont bafoués? Avec le temps, certaines organisations se placent dans un cercle vicieux. Elles n'ont pas la moindre reconnaissance du groupe adverse - généralement représenté par un État autoritaire, voire sanguinaire — mais ne la souhaitent pas non plus puisqu'elles se considèrent dorénavant comme au-dessus de la mêlée, comme étant une avant-garde de combattants ou les derniers résistants. Peu à peu, elles perdent ce lien fondamental avec les communautés existantes qu'elles souhaitent représenter. Une arme redoutable se développe progressivement au fond d'elles-mêmes : un moi collectif suridéalisé et rattaché à une communauté fictive. La dimension sociopsychologique prend alors tout son sens. Au sein d'une organisation radicale armée, une vision amplifiée de l'adversaire peut empêcher toute réconciliation avec un État qui, par ailleurs, est sans pardon à l'égard de ce genre d'opposition. Cette situation extrême est bien différente de celle qui s'est finalement produite au Chiapas lors de la rébellion des sans-visage ${ }^{18}$.

\section{RÉSUMÉ}

L'auteur analyse le mouvement zapatiste au Chiapas afin de connaître les ajustements cognitifs

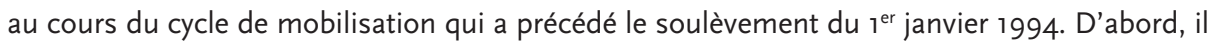
souligne le rôle de facteurs classiques mis en évidence par la perspective de la mobilisation des ressources : la structure d'occasions politiques et l'organisation. Ces facteurs ont contribué au développement d'une structure de mobilisation bien spécifique dans la Selva, une région reconnue pour être le bastion des zapatistes. Enfin, l'analyse de témoignages d'Indiens zapatistes révèle la présence de deux processus sociopsychologiques au sein du mouvement, et ce avant la rébellion : la revalorisation du soi collectif et l'amplification des stéréotypes à l'égard des ladinos (non-Indiens). L'Église catholique a contribué à revaloriser le soi collectif. L'amplification des stéréotypes est attribuée à l'influence des guérilleros des Fuerzas de Liberación Nacional (FLN) et, indirectement, à celle de l'autoritarisme de l'État.

\section{SUMMARY}

The author analyses the Zapatist movement in the Chiapas to study the cognitive adjustments that occurred during the cycle of mobilization that preceded the uprising on January 1st, 1994. First, he stresses the role of classic factors revealed by the prospect of the mobilization of resources : the structure of political opportunities and organization. These factors contributed to the development of a very specific mobilization structure in the Selva, an area known to be the Zapatist bastion. Second, the analysis of testimonies from Zapatist Indians reveals the presence of two socio-psychological processes within the movement, even before the rebellion : the reassertion of their sense of collective identity and the growing spread of stereotypes regarding the ladinos (non-Indians). The Catholic Church helped to promote the collective identity. The spread of stereotypes is attributed to the influence of the FLN guerilleros (Fuerzas de Liberacion Nacional) and indirectly to the authoritarianism of the State.

18. Dans certains communiqués, l'EZLN se définit lui-même en utilisant cette expression «les sansvisage». 


\section{RESUMEN}

El autor analiza el movimiento zapatista a Chiapas con el fin de conocer los ajustes cognoscitivos durante el ciclo de movilización que precedió el levantamiento del 1 de enero de 1994. En primer lugar, él destaca el papel de factores clásicos puestos de relieve por la perspectiva de la movilización de los recursos : la estructura de ocasiones políticas y la organización. Estos factores contribuyeron al desarrollo de una estructura de movilización bien específica en el selva, una región reconocida por ser el bastión de los zapatistas. Por último, el análisis de testimonios de indios zapatistas revela la presencia de dos procesos sociopsicológicos en el movimiento, y esto antes de la rebelión : la revalorización del sí colectivo y la amplificación de los estereotipos al respeto de los ladinos (no indios). La Iglesia Católica contribuyó a revalorizar el sí colectivo. La amplificación de los estereotipos se asigna a la influencia de los guerrilleros de las Fuerzas de Liberación Nacional (FLN) e indirectamente del autoritarismo del Estado.

\section{BIBLIOGRAPHIE}

AL-Berry, K. (2002), La Terre est plus belle que le paradis, Paris, JC Lattès.

Amnistie internationale (1986), Mexique : violation des droits de l'homme en zones rurales, Paris, efai. ARIAS, J. (1994), «Nuestra batalla para pertenecernos a nosotros mismos», in Maria Luisa Armendáriz (dir.), Chiapas, una radiografía, Mexico, Fondo de Cultura Económica, p. 198-210.

Chazel, F. (1997), «Les ajustements cognitifs dans les mobilisations collectives», in F. Chazel, R. Boudon et

A. Bouvier (dir.), Cognition et sciences sociales, Paris, Presses Universitaires de France, p. 193-206.

Cormier, G. (2002), La rébellion des sans-visage : analyse de l'émergence du mouvement zapatiste au Chiapas (Mexico), thèse de sociologie, Paris, Université de Paris Iv-Sorbonne.

Couffignal, G. (1988), «Le Parti révolutionnaire institutionnel mexicain : Crépuscule d'une hégémonie?», Problèmes d'Amérique latine, $\mathrm{n}^{\circ}$ 4858, p. 3-24.

De La Grange, B. et M. Rico (1998), Sous-commandant Marcos : La géniale imposture, Paris, Plon.

Ferree, M. M. et F. D. Miller (1985), «Mobilization and Meaning: Toward an Integration of Social Psychological and Ressource Perspectives on Social Movements», Sociological Inquiry, vol. 55, $\mathrm{n}^{\circ} 1$, p. 38-61.

Gonzalez Casanova, P. (1996), «Les causes de la révolte chiapanèque», in Luis E. Gomez (dir.), Mexique: de Chiapas à la crise financière, Paris, L'Harmattan, p. 131-153.

Harvey, N. (2000), La rebelión de Chiapas, Mexico, Era.

Hewstone, M. (1989), Causal Attribution : From Cognitive Processes to Collective Beliefs, Oxford, Blackwell. HogG, M. A. et D. Abrams (1988), Social Identifications : A Social Psychology of Intergroup Relations and Group Processes, Londres, Routledge.

Krauze, E. (1999), «El profeta de los Indios», Letras libres, nº 1, p. 10-18, 86-97.

Le Bot, Y. (1997), Le rêve zapatiste, Paris, Seuil.

Legorreta Díaz, M. et Del C. (1998), Religión, política y guerrilla en Las Cañadas de la Selva Lacandona, Mexico, Cal y Arena.

Leyva Solano, X. et G. Ascencio Franco (1996), Lacandonia al filo del agua, Mexico, Centro de Investigaciones y Estudios Superiores en Antropología Social.

Mattiace, S. L. (2001), «Regional Renegotiations of Space : Tojolabal Ethnic Identity in Las Margaritas, Chiapas», Latin American Perspectives, 117, vol. 28, $\mathrm{n}^{\circ}$ 2, p. 73-97.

McAdam, D. (1982), Political Process and The Development of Black Insurgency 1930-1970, Chicago, University of Chicago Press.

McCarthy, J.D. et M.N. ZaLd (1977), «Resource Mobilization and Social Movements : A Partial Theory», American Journal of Sociology, vol. 82, p. 1212-1241.

Mead, G. H. (1967), Mind, Self and Society, Chicago, University of Chicago Press. 
Meyer, J. (2000), Samuel Ruiz en San Cristóbal, Mexico, Tusquets.

Oberschall, A. (1973), Social Conflict and Social Movements, Englewood Cliffs, Prentice-Hall.

Pettigrew, T.F. (1979), «The Ultimate Attribution Error : Extending Allport's Cognitive Analysis of Prejudice», Personality and Social Psychology Bulletin, vol. 5, p. 461-476.

PorTa (della), D. (1992), «The Research on Individual Motivations in Underground Political Organizations », International Social Movement Research, vol. 4, p. 3-28.

Ramonet, I. (2001), Marcos : la dignité rebelle, Paris, Galilée.

Rus, J. (1995), «La Comunidad revolucionaria institucional», in J. P. Viqueira et M. Humberto Ruz, Chiapas : Los rumbos de otra historia, Mexico, Universidad Nacional Autónoma de México.

SNow, D. A., E. B. Rochford Jr., S. K. Worden et R. D. Benford (1986), «Frame Alignment Processes, Micromobilization, and Movement Participation", American Sociological Review, vol. 51, p. 464-481.

Tajfel, H. et J.C. Turner (1979), «An Integrative Theory of Intergroup Conflict», in W.G. Austin et S. Worchel (éd.), The Social Psychology of Intergroup Relations, Monterey, Brooks Cole.

Tarrow, S. (1998), Power in Movement: Social Movements and Contentious Politics, Cambridge, Cambridge University Press.

Tello Diaz, C. (200o), La rebelión de las Cañadas, Mexico, Cal y Arena.

Tilly, C. (1978), From Mobilization to Revolution, Reading, Addison-Wesley.

Triandis, H. C. (1989), «The Self and Social Behavior in Differing Cultural Contexts», Psychological Review, vol. $96, \mathrm{n}^{\circ} 3$, p. 506-520.

Womack Jr., J. (1999), Rebellion in Chiapas, New York, The New Press. 